\title{
Prognostic impact of restored sinus rhythm in patients with sepsis and new-onset atrial fibrillation
}

\author{
Wen Cheng Liu', Wen Yu Lin', Chin Sheng Lin', Han Bin Huang², Tzu Chiao Lin', Shu Meng Cheng',
} Shih Ping Yang ${ }^{1}$, Jung Chung $\operatorname{Lin}^{3}$ and Wei Shiang Lin ${ }^{1 *}$

\begin{abstract}
Background: New-onset atrial fibrillation (NeOAF) is a common type of tachyarrhythmia in critically ill patients and is associated with increased mortality in patients with sepsis. However, the prognostic impact of restored sinus rhythm (SR) in septic patients with NeOAF remains unclear.

Methods: A total of 791 patients with sepsis, who were admitted to a medical intensive care unit from January 2011 to January 2014, were screened. NeOAF was detected by continuous electrocardiographic monitoring. Patients were categorized into three groups: no NeOAF, NeOAF with restored SR (NeOAF to SR), and NeOAF with failure to restore SR (NeOAF to atrial fibrillation (AF)). The endpoint of this study was in-hospital mortality. Patients with preexisting AF were excluded.

Results: We reviewed the data of 503 eligible patients, including 263 patients with no NeOAF and 240 patients with NeOAF. Of these 240 patients, SR was restored in 165 patients, and SR could not be restored in 75 patients. The NeOAF to AF group had the highest in-hospital mortality rate of $61.3 \%$ compared with the NeOAF to SR and no NeOAF groups (26.1\% and $17.5 \%$, respectively). Moreover, multivariate logistic regression analysis revealed that failure of restored SR was independently associated with increased in-hospital mortality in patients with sepsis and $\mathrm{NeOAF}$.

Conclusions: Failure to restore a sinus rhythm in patients with new-onset atrial fibrillation may be associated with increased in-hospital mortality in patients with sepsis. Further prospective studies are needed to clarify the effects of restoration of sinus rhythm on survival in patients with sepsis and new-onset atrial fibrillation.
\end{abstract}

Keywords: New-onset atrial fibrillation, Sepsis, Mortality

\section{Background}

Atrial fibrillation (AF) is the most common sustained cardiac arrhythmia in critically ill patients $[1,2]$. In past decades, increased attention has been paid to new-onset atrial fibrillation (NeOAF) in patients undergoing cardiac or noncardiac surgery, patients with major trauma, and critically ill patients, and it is associated with poor prognosis [3-10].

\footnotetext{
* Correspondence: wslin545@ms27.hinet.net

'Division of Cardiology, Department of Internal medicine, Tri-Service General Hospital, National Defense Medical Center, No. 325, Section 2, Cheng-Gong Road, Neihu 114, Taipei, Taiwan

Full list of author information is available at the end of the article
}

Sepsis, a potentially life-threatening organ dysfunction syndrome caused by a dysregulated host response to infection, is one of the leading causes of death worldwide. On the basis of disease severity, it can be categorized into sepsis and septic shock. Septic shock is defined by requirement for vasopressors to maintain a mean arterial pressure of $65 \mathrm{mmHg}$ or greater and serum lactate level greater than $2 \mathrm{mmol} / \mathrm{L}$ in the absence of hypovolemia [11-13]. In recent studies, NeOAF has been shown to be prevalent in patients with sepsis and to be associated with longer hospital stay and increased morbidity and mortality [14-24].

A longer AF burden leads to worsening cardiac effects and makes sepsis treatment more difficult. A previous 
study found that management with electrical cardioversion or pharmacological treatment for NeOAF may improve outcomes in critically ill patients [18]. It remains unclear whether restored sinus rhythm (SR) in patients with sepsis and NeOAF is associated with favorable prognosis. Therefore, among patients with sepsis, this study investigated the impact on in-hospital mortality of restoration of SR in patients with $\mathrm{NeOAF}$ in comparison with failure to restore SR in those with NeOAF and without NeOAF.

\section{Methods}

\section{Study population}

This single-center, retrospective, comparative cohort study was conducted at Tri-Service General Hospital, National Defense Medical Center, and involved screening of consecutive adults who were admitted to a medical intensive care unit (ICU) with a diagnosis of sepsis or septic shock from January 2011 to January 2014. The institutional review board of the center approved the study with a protocol number of 2-104-05-003. Informed consent was waived due to the observational design.

Previous medical records and a 12-lead electrocardiogram (ECG) were fully evaluated by chart review. Preexisting AF and arrhythmia other than AF were excluded initially. Then, individuals meeting any of the following criteria were excluded: (1) age older than 90 years; (2) ICU stay less than 3 days; (3) missing or incomplete clinical data or ECG; or (4) major surgery within 2 weeks prior to this admission. A flowchart of the enrollment of the study population is shown in Fig. 1.

Medical records were reviewed to collect information on baseline characteristics including demographic data, laboratory parameters, and the underlying comorbidities of hypertension, diabetes mellitus (DM), coronary artery

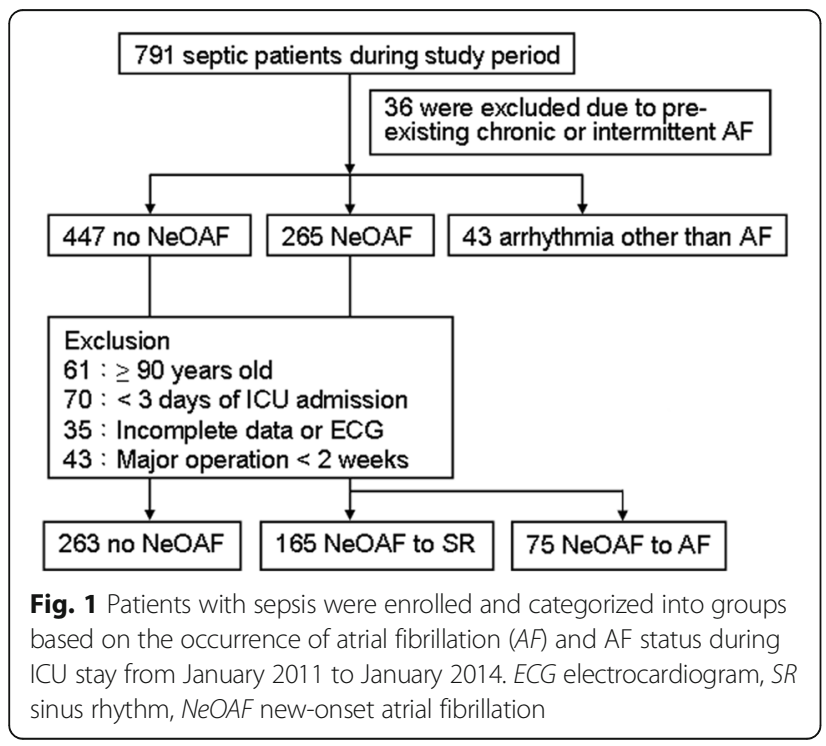

disease (CAD), heart failure, cerebrovascular disease (CVD), chronic obstructive pulmonary disease (COPD), chronic kidney disease (CKD), and prior thyroid disease. Prior medication use, including beta-blockers or nondihydropyridine calcium channel blockers (non-DHP CCBs) was evaluated. Transthoracic echocardiographic findings were assessed on ICU admission with a focus on left atrium diameter (LAD), left ventricular end-diastolic dimension (LVEDD), left ventricular end-systolic dimension (LVESD), and left ventricular ejection fraction (LVEF).

\section{Diagnosis of sepsis and its severity}

Sepsis and septic shock were defined based on the The Third International Consensus Definitions for Sepsis and Septic shock (Sepsis-3) [13]. The management of sepsis was based on early goal-directed therapy recommended in the guidelines of the Surviving Sepsis Campaign $[11,12]$.

According to Surviving Sepsis Campaign recommendations, information on infection sites and sepsisinduced acute organ dysfunction, including neurologic, circulatory, respiratory, renal, hepatic, and hematologic dysfunction was collected. Scores of severity-of-disease classification systems including the Acute Physiology and Chronic Health Evaluation II (APACHE II) and Sequential Organ Failure Assessment (SOFA) were recorded for each individual on ICU admission $[25,26]$.

\section{Detection and management of $\mathrm{NeOAF}$}

During ICU admission, all patients received continuous electrocardiographic monitoring. The 12-lead ECGs were obtained when deemed clinically appropriate by the critical care team. The diagnosis of AF, defined as the absence of $\mathrm{P}$ waves and irregular ventricular activity lasting for more than 30 seconds, was confirmed by clinicians. After NeOAF was detected, the decision of electrical cardioversion, pharmacological treatment, or a wait-and-see strategy for NeOAF was made by the responsible clinicians based on the patient's hemodynamic status and contraindication for antiarrhythmic agents.

Failure to restore SR in patients with NeOAF was defined as persistent or recurrent AF 7 days after the onset of NeOAF and was referred to as NeOAF to AF. The restoration of SR in patients with $\mathrm{NeOAF}$ is referred to as NeOAF to SR. Patients with NeOAF who died within 7 days after admission was categorized as the indicated group based on the final rhythm before death. The endpoint of this study was in-hospital mortality, which was confirmed by records of the death note.

\section{Statistical analyses}

Statistical analyses were performed using the SPSS software package (version 17.0; SPSS, Chicago, IL, USA), 
and differences were considered statistically significant when the $P$ value was $<0.05$. Continuous variables are presented as mean and standard deviation. Categorical variables are presented as the number of patients and the corresponding percentage. The differences in the characteristics of the groups were assessed using the unpaired two-tailed Student $t$ test or one-way analysis of variance (ANOVA) for continuous variables and the chisquare and Fisher exact tests for nominal variables.

The significant variables $(P<0.05)$ associated with the individual AF status in patients were identified. Backward stepwise logistic regression analysis was conducted to evaluate the significant predictors of $\mathrm{NeOAF}$ and NeOAF to AF. To evaluate the association between the NeOAF status and in-hospital mortality, we constructed two regression models. Model 1 was the univariate logistic regression analysis of the AF status. Model 2 was the multivariate logistic regression analysis after adjustment for significant confounding factors, which were selected based on the criteria of being associated with exposure, associated with outcomes, and not intermediate variables between exposure and outcome [27]. A Cox proportional hazard model with time-varying exposure was constructed to confirm the associations between individual AF status and in-hospital mortality.

\section{Results}

\section{Study population and incidence of NeOAF}

A total of 791 patients were screened. Of these patients, 36 and 43 patients were excluded due to pre-existing AF and arrhythmia other than AF, respectively. The prevalence of pre-existing AF was $4.5 \%$ (36/791). The incidence of NeOAF was 35.1\% (265/755). After excluding patients older than 90 years, with less than 3 days of ICU stay, missing or incomplete data, or having had major surgery within 2 weeks of sepsis, 503 eligible patients were evaluated based on the different AF status. Among these, 263 patients had no AF and 240 patients developed NeOAF after ICU admission. SR was restored in 165 patients (NeOAF to SR) and SR could not be restored in 75 patients (NeOAF to AF) as shown in Fig. 1.

\section{Baseline characteristics of patients with sepsis and different AF status}

The baseline characteristics of patients with sepsis and different AF status are compared in Table 1. Patients in the NeOAF to SR and NeOAF to AF groups were significantly older than those in the no NeOAF group. Moreover, a higher prevalence of hypertension, heart failure, CAD, and CVD was observed in patients with NeOAF compared with those without NeOAF. No significant differences were observed in COPD, DM, uremia, and thyroid disorder between these three groups. Regarding prior medication use before ICU admission, patients with the NeOAF to AF group had lower beta-blocker use compared with those with the NeOAF to SR group.

Table 2 shows the laboratory and echocardiographic results in patients with sepsis and different AF status. All the laboratory data and examinations were performed at the beginning of ICU admission. No statistically significant differences were observed in serum white blood cell (WBC) count, electrolytes, albumin, or B-type natriuretic peptide (BNP) levels among the three

Table 1 Baseline characteristics of patients with sepsis and different AF status

\begin{tabular}{|c|c|c|c|c|}
\hline \multirow[t]{2}{*}{ Characteristics } & \multicolumn{3}{|c|}{ Overall patients $(n=503)$} & \multirow[t]{2}{*}{$P$ value } \\
\hline & No NeOAF $(n=263)$ & NeOAF to SR $(n=165)$ & NeOAF to $\mathrm{AF}(n=75)$ & \\
\hline Age, years & $69.5 \pm 15.6$ & $77.8 \pm 10.3 \dagger$ & $76.2 \pm 11.0^{a}$ & $<0.01$ \\
\hline Male, $n(\%)$ & $174(66.2)$ & $90(54.5) \dagger$ & $46(61.3)$ & 0.06 \\
\hline \multicolumn{5}{|l|}{ Comorbidities, n (\%) } \\
\hline Hypertension & $145(55.1)$ & $111(67.3) \dagger$ & $44(58.7)$ & 0.04 \\
\hline Heart failure & $25(9.5)$ & $35(21.2) \dagger$ & $15(20.0)^{a}$ & $<0.01$ \\
\hline Coronary artery disease & $90(34.2)$ & $70(42.4)$ & $37(49.3)^{a}$ & 0.04 \\
\hline Cerebrovascular disease & $56(21.3)$ & $53(32.1) \dagger$ & $23(30.7)$ & 0.03 \\
\hline COPD & $52(19.8)$ & $28(17.0)$ & $12(16.0)$ & 0.66 \\
\hline Diabetes mellitus & $97(36.9)$ & $65(39.4)$ & $21(28.0)$ & 0.23 \\
\hline Uremia & $18(6.8)$ & $17(10.3)$ & $9(12.0)$ & 0.26 \\
\hline Thyroid disorder & $10(3.8)$ & $10(6.1)$ & $6(8.0)$ & 0.29 \\
\hline \multicolumn{5}{|l|}{ Prior medication, $n$ (\%) } \\
\hline Beta blocker & $29(11.0)$ & $27(16.4)$ & $4(5.3)^{b}$ & 0.04 \\
\hline Calcium channel blocker & $9(3.4)$ & $10(6.1)$ & $2(2.7)$ & 0.32 \\
\hline
\end{tabular}


Table 2 Laboratory findings and echocardiography index at admission in patients with sepsis and different AF status

\begin{tabular}{|c|c|c|c|c|}
\hline & \multicolumn{3}{|c|}{ Overall patients $(n=503)$} & \multirow[t]{2}{*}{$P$ value } \\
\hline & No NeOAF $(n=263)$ & NeOAF to SR $(n=165)$ & $\mathrm{NeOAF}$ to $\mathrm{AF}(n=75)$ & \\
\hline \multicolumn{5}{|l|}{ Laboratory } \\
\hline WBC $\left(\times 10^{3} / L\right)$ & $13.47 \pm 8.73$ & $13.43 \pm 7.23$ & $15.39 \pm 11.73$ & 0.22 \\
\hline CRP (mmol/L) & $12.8 \pm 10.9$ & $10.0 \pm 9.9+$ & $11.2 \pm 8.5$ & 0.02 \\
\hline $\mathrm{Na}^{+}(\mathrm{mmol} / \mathrm{L})$ & $135.6 \pm 8.5$ & $135.7 \pm 10.1$ & $136.7 \pm 7.8$ & 0.64 \\
\hline $\mathrm{K}^{+}(\mathrm{mmol} / \mathrm{L})$ & $4.1 \pm 1.0$ & $4.2 \pm 1.0$ & $4.1 \pm 0.9$ & 0.46 \\
\hline Free $\mathrm{Ca}^{2+}(\mathrm{mmol} / \mathrm{L})$ & $4.23 \pm 0.31$ & $4.35 \pm 0.46$ & $4.17 \pm 0.68$ & 0.46 \\
\hline Albumin (g/dL) & $2.7 \pm 0.6$ & $2.7 \pm 0.6$ & $2.6 \pm 0.6$ & 0.37 \\
\hline BNP (pg/ml) & $918.6 \pm 1152.2$ & $1131.3 \pm 1325.6$ & $1102.4 \pm 1418.1$ & 0.58 \\
\hline Tr-l (ng/ml) & $1.09 \pm 5.92$ & $1.98 \pm 5.95$ & $3.71 \pm 13.42^{a}$ & 0.04 \\
\hline \multicolumn{5}{|l|}{ Echocardiography } \\
\hline LAD (mm) & $36.5 \pm 7.4$ & $38.4 \pm 7.2+$ & $40.5 \pm 7.2^{a}$ & $<0.01$ \\
\hline $\operatorname{LVEDD}\left(\mathrm{mm}^{3}\right)$ & $45.7 \pm 8.0$ & $46.1 \pm 7.7$ & $46.6 \pm 7.5$ & 0.73 \\
\hline $\operatorname{LVESD}\left(\mathrm{mm}^{3}\right)$ & $30.3 \pm 8.0$ & $30.9 \pm 7.3$ & $32.3 \pm 8.4$ & 0.22 \\
\hline LVEF < 50\%, n (\%) & $31(13.7)$ & $29(18.2)$ & $18(27.7) \dagger$ & 0.03 \\
\hline
\end{tabular}

NeOAF new-onset atrial fibrillation, $A F$ atrial fibrillation, $S R$ sinus rhythm, WBC white blood cell count, CRP C-reactive protein, $B N P$ B-type natriuretic peptide, Tr- 1 troponin I, $L A D$ left atrium diameter, $L V E D D$ left ventricular end diastolic diameter, $L V E S D$ left ventricular end systolic diameter, $L V E F$ left ventricular ejection fraction. ${ }^{a} P<0.05$ vs. no NeOAF

groups. However, C-reactive protein (CRP) was lowest in the NeOAF to SR group than in the no NeOAF and NeOAF to AF groups $(10.0 \pm 9.9,12.8 \pm 10.9$ and $11.2 \pm$ $8.5 \mathrm{mmol} / \mathrm{L}$, respectively). Troponin-I was highest in the $\mathrm{NeOAF}$ to AF group than in the no NeOAF and NeOAF to SR groups. On echocardiography, both the NeOAF to $\mathrm{SR}$ and NeOAF to AF group had a larger left atrial diameter than the no NeOAF group. Left ventricular systolic dysfunction was more prevalent in patients with NeOAF to AF.

\section{Sepsis severity in patients with different AF status}

The most common infection site of sepsis in our study population was the respiratory tract, followed by the urinary tract and intra-abdominal sites (Table 3). Patients with NeOAF had significantly high SOFA and APACHE II scores, which are severity indices of sepsis. The no NeOAF, NeOAF to SR, and NeOAF to AF groups had SOFA scores of $7.0 \pm 3.2,7.6 \pm 3.0$, and $9.3 \pm$ 3.2 and APACHE II scores of $21.6 \pm 5.5,22.8 \pm 5.8$, and $24.6 \pm 6.1$, respectively. Dopamine and norepinephrine were more commonly used in the NeOAF to AF group.

\section{Management of NeOAF in patients with sepsis}

We reviewed 240 eligible patients with NeOAF. Among these 240 patients, SR was restored in 165 patients (NeOAF to SR), and SR could not be restored in 75 patients (NeOAF to AF). Treatments received by patients with NeOAF are shown in Table 4. Beta-blockers (36.7\%) and amiodarone (33.3\%) were the most commonly used in patients with NeOAF and sepsis, followed by non-DHP CCBs and digitalis glycosides. Electrical cardioversion was performed in only eight patients. There were no significant differences in pharmacological therapies and electrical cardioversion between the $\mathrm{NeOAF}$ to SR and NeOAF to AF groups.

\section{Clinical impact of rhythm control in patients with sepsis and NeOAF}

Patients with sepsis and NeOAF had a longer ICU stay (NeOAF to SR group: $16.7 \pm 13.6$; NeOAF to AF group: $17.3 \pm 23.3$ days) than those without NeOAF (11.4 \pm 11.1 days). Moreover, the NeOAF to AF group had the highest in-hospital mortality rate of $61.3 \%$ (46/75) compared with the rate of $26.1 \%(43 / 165)$ in the NeOAF to SR group and of $17.5 \%(46 / 263)$ in the no NeOAF group $(P<0.01)$ as shown in Fig. 2.

Based on the data in Tables 1, 2, 3, and 4, the significant variables were selected associated with different AF status as follows: age; hypertension; heart failure; CAD; CVD; prior beta-blocker use; CRP; Troponin-I; LA dimension; LVEF; SOFA scores; APACHE II scores; neurological, circulation, or hepatic failure; renal dysfunction; dopamine use; and norepinephrine use. The logistic regression models were constructed to analyze the singificant predictors of NeOAF and NeOAF to AF in patients with sepsis. For all patients with $\mathrm{NeOAF}$, these predictors included age, heart failure, CVD, and SOFA scores. For patients with NeOAF to $\mathrm{AF}$, these predictors included age, LA dimension, APACHE II scores, hepatic dysfunction, dopamine use, and norepinephrine use.

Regarding the association between different AF status and in-hospital mortality, the univariate logistic regression 
Table 3 Disease severity index of sepsis in patients with various AF statuses

\begin{tabular}{|c|c|c|c|c|}
\hline & \multicolumn{3}{|c|}{ Overall patients $(n=503)$} & \multirow[t]{2}{*}{$P$ value } \\
\hline & No NeOAF $(n=263)$ & NeOAF to SR $(n=165)$ & NeOAF to $\mathrm{AF}(n=75)$ & \\
\hline \multicolumn{5}{|l|}{ Infection site, $n$ (\%) } \\
\hline Respiratory tract & $168(63.9)$ & $112(67.9)$ & $48(64.0)$ & \\
\hline Urinary tract & $57(21.7)$ & $35(21.2)$ & $14(18.7)$ & \\
\hline Gastrointestinal & $23(8.7)$ & $9(5.5)$ & $5(6.7)$ & \\
\hline Others & $15(5.7)$ & $9(5.5)$ & $8(10.7)$ & \\
\hline SOFA score & $7.0 \pm 3.2$ & $7.6 \pm 3.0$ & $9.3 \pm 3.2^{b c}$ & $<0.01$ \\
\hline APACHE I| score & $21.6 \pm 5.5$ & $22.8 \pm 5.8$ & $24.6 \pm 6.1^{b}$ & $<0.01$ \\
\hline Total organ failure ${ }^{a}, n$ & $2(1-3)$ & $2(1-3)$ & $3(2-4)$ & \\
\hline Neurologic failure & $87(33.1)$ & $49(29.7)$ & $39(52.0)^{b c}$ & $<0.01$ \\
\hline Circulatory failure & $118(44.9)$ & $82(49.7)$ & $57(76.0)^{b c}$ & $<0.01$ \\
\hline Respiratory failure & $229(87.1)$ & $150(90.9)$ & $71(94.7)$ & 0.13 \\
\hline Hepatic failure & $8(3.0)$ & $4(2.4)$ & $8(10.7)^{\mathrm{bc}}$ & $<0.01$ \\
\hline Renal failure & $86(32.7)$ & $65(39.4)$ & $39(52.0)^{b}$ & $<0.01$ \\
\hline Hematologic failure & $9(3.4)$ & $5(3.0)$ & $4(5.3)$ & 0.66 \\
\hline \multicolumn{5}{|l|}{ Vasopressor use, $n(\%)$} \\
\hline Dopamine use & $95(36.3)$ & $64(38.8)$ & $49(65.3)^{\mathrm{bc}}$ & $<0.01$ \\
\hline Norepinephrine use & $80(30.4)$ & $58(35.2)$ & $48(64.0)^{\mathrm{bc}}$ & $<0.01$ \\
\hline \multicolumn{5}{|l|}{ Intervention, n (\%) } \\
\hline Ventilator use & $221(84.0)$ & $143(86.7)$ & $69(92.0)$ & 0.21 \\
\hline New-onset dialysis & $50(19.0)$ & $35(21.2)$ & $19(25.3)$ & 0.48 \\
\hline
\end{tabular}

NeOAF new-onset atrial fibrillation, $A F$ atrial fibrillation, SR sinus rhythm SOFA Sequential Organ Failure Assessment score, APACHE II Acute Physiology and Chronic Health Evaluation II. ${ }^{\mathrm{a} T}$ Total organ failure is presented as median and interquartile range. ${ }^{\mathrm{b}} P<0.05$ vs. no NeOAF. ${ }^{C} P<0.05$ vs. NeOAF to SR

analysis showed that NeOAF to AF was significantly associated with increased in-hospital mortality compared to NeOAF to $\mathrm{SR}$ and no NeOAF $(\mathrm{OR}=4.50$, 95\% $\mathrm{CI}=2.52-8.04 ; P<0.01$ and $\mathrm{OR}=7.48,95 \% \mathrm{CI}$ $=4.26-13.14 ; P<0.01)$ (Table 5). The multivariate logistic regression analysis, which was adjusted for the significant confounding factors including: age; CAD; LVEF; SOFA scores; neurological, circulation, hepatic, or renal dysfunction; and dopamine and norepinephrine use, revealed that $\mathrm{NeOAF}$ to AF itself was an independent risk factor for inhospital mortality in patients with sepsis compared to NeOAF to SR and no NeOAF (OR $=2.22$, 95\% CI $=1.02-$ 4.83; $P<0.05$ and $\mathrm{OR}=3.31,95 \% \mathrm{CI}=1.54-7.13 ; P<0.01$ ).
Moreover, Cox proportional hazard models were applied to examine the association between different AF status and inhospital mortality. There were some differences compared with the logistic regression model, especially in the association between NeOAF to AF or no NeOAF and in-hospital mortality (Additional file 1: Table S1). However, the associations between NeOAF to AF or NeOAF to SR and inhospital mortality were similar $(P<0.05)$.

\section{Discussion \\ Main findings}

In this study, we demonstrated that NeOAF in patients with sepsis was associated with an increased risk of in-

Table 4 Therapeutic interventions in patients with sepsis and NeOAF

\begin{tabular}{|c|c|c|c|c|}
\hline & \multicolumn{3}{|c|}{ NeOAF patients $(n=240)$} & \multirow[t]{2}{*}{$P$ value } \\
\hline & Overall $(n=240)$ & NeOAF to SR $(n=165)$ & NeOAF to $\mathrm{AF}(n=75)$ & \\
\hline \multicolumn{5}{|l|}{ Pharmacological, $n$ (\%) } \\
\hline Amiodarone & $80(33.3)$ & $52(31.5)$ & $28(37.3)$ & 0.38 \\
\hline Beta-blockers & $88(36.7)$ & $67(40.6)$ & $21(28.0)$ & 0.06 \\
\hline Non-DHP CCBs ${ }^{\mathrm{a}}$ & $66(27.5)$ & $47(28.5)$ & $19(25.3)$ & 0.61 \\
\hline Digoxin glycosides & $27(11.3)$ & $15(9.1)$ & $12(16.0)$ & 0.12 \\
\hline Electrical cardioversion, $n$ (\%) & $8(3.3)$ & $4(2.4)$ & $4(5.3)$ & 0.25 \\
\hline
\end{tabular}

NeOAF new-onset atrial fibrillation, $A F$ atrial fibrillation, $S R$ sinus rhythm. ${ }^{a}$ Only indicates non-dihydropyridine calcium channel blockers (non-DHP CCBs) 


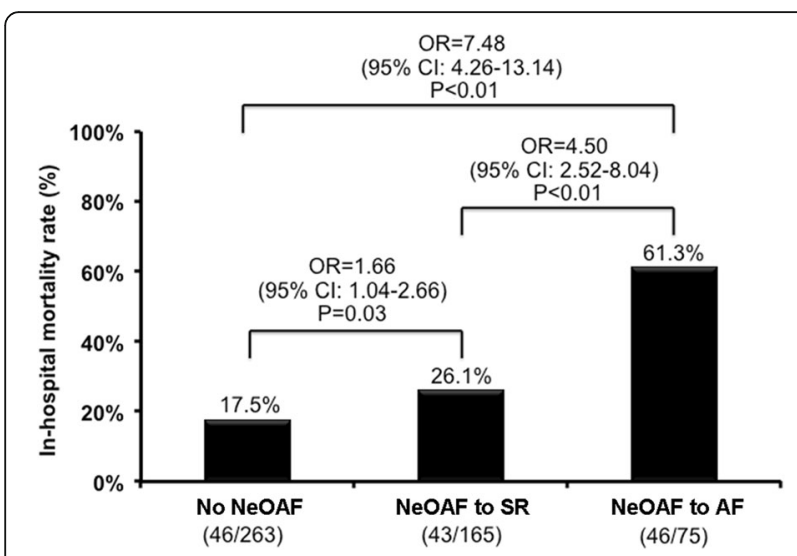

Fig. 2 In-hospital mortality in patients with sepsis and different AF status. NeOAF new-onset atrial fibrillation, AF atrial fibrillation, $S R$ sinus rhythm

hospital mortality. Baseline organ dysfunction, especially neurologic, circulatory, hepatic, or renal dysfunction (which are significant confounders for in-hospital mortality and NeOAF status in patients with sepsis), was statistically different in the three groups. Intriguingly, patients with $\mathrm{NeOAF}$ in whom SR could not be restored (as NeOAF to $\mathrm{AF}$ ) has 2.22-fold higher risks of in-hospital mortality than those in whom SR was restored (as NeOAF to SR) and 3.31-fold higher risks of in-hospital mortality than those without NeOAF (as no NeOAF). In addition, in-hospital mortality in patients with NeOAF and restored SR was not statistically different compared with patients with no $\operatorname{NeOAF}(P=0.22,95 \% \mathrm{CI}=0.79-2.83)$.

Previous research suggests that the prevalence of preexisting AF among hospitalized patients ranges from 2 to $15 \%$. In our study, the prevalence of pre-existing AF was $4.5 \%$. Pre-existing AF was evaluated by previous medical records and 12-lead ECG at admission, which may underestimate the prevalence of AF in patients with unrecognized paroxysmal AF. Moreover, the incidence

Table 5 Association between different AF status and in-hospital mortality

\begin{tabular}{llll}
\hline Model & OR & $95 \%$ Cl of OR & $P$ value \\
\hline Model $1^{\text {a }}$ & & & \\
NeOAF to SR vs. no NeOAF & 1.66 & $1.04-2.66$ & 0.03 \\
NeOAF to AF vs. no NeOAF & 7.48 & $4.26-13.14$ & $<0.01$ \\
NeOAF to AF vs. NeOAF to SR & 4.50 & $2.52-8.04$ & $<0.01$ \\
Model 2 & & & \\
NeOAF to SR vs. no NeOAF & 1.49 & $0.79-2.83$ & 0.22 \\
NeOAF to AF vs. no NeOAF & 3.31 & $1.54-7.13$ & $<0.01$ \\
NeOAF to AF vs. NeOAF to SR & 2.22 & $1.02-4.83$ & 0.045 \\
\hline
\end{tabular}

NeOAF new-onset atrial fibrillation, $A F$ atrial fibrillation, $S R$ sinus rhythm, $O R$ odds ratio, $\mathrm{Cl}$ confidence interval. ${ }^{\mathrm{A}}$ Unadjusted model. ${ }^{\mathrm{b}}$ Adjusted for age, coronary artery disease, left ventricular ejection fraction, Sequential Organ Failure Assessment score, neurologic failure, circulatory failure, hepatic failure, renal failure, dopamine use, and norepinephrine use of NeOAF was $35.1 \%$ in this study, which is higher than previous studies ranging from 10 to $46 \%$ [22]. It is probably due to higher disease severity, organ dysfunction and frequent vasopressor use among the enrolled patients.

\section{Association between sepsis and NeOAF}

According to the mechanism of NeOAF proposed by most studies, NeOAF in patients with sepsis is attributable to sepsis-related inflammatory states. Basically, systemic inflammation can alter atrial electrophysiology and structural substrates, leading to increased vulnerability to AF. Complex inflammatory pathways affect myolysis, cardiomyocyte apoptosis, and the activation of fibrotic pathways through fibroblasts, transforming growth factor- $\beta$, and matrix metalloproteases, contributing to structural remodeling of the atria [28, 29]. In addition, sepsis-related micro-abscess formation and autonomic dysfunction may be also responsible for the potential mechanisms for the occurrence of $\mathrm{NeOAF}[30,31]$.

The clinical management of patients with sepsis is also associated with increased risks of NeOAF. Previous studies suggest that fluid resuscitation and vasopressor use in response to hypotension greatly contribute to the development of NeOAF [24, 32]. Increased net positive fluid resuscitation may result in an acute increase in left ventricular end-diastolic pressure and subsequent left atrial stretch, providing an anatomical substrate for the occurrence of AF. Vasopressor agents, particularly those with $\beta$-adrenergic effects, may also play a direct role in triggering $\mathrm{NeOAF}[14,18,24,33]$.

Serum CRP, an acute-phase reactant, has been considered a clinical indicator of inflammation. Hu et al. demonstrated a positive relationship between elevated CRP and the development and maintenance of AF [29]. AF is more common in patients with high CRP than in those with low CRP. Aviles et al. also reported that baseline CRP can be used to predict the risk of AF in the general population [34]. In patients with sepsis, Meierhenrich et al. demonstrated continuous increments in CRP before the onset of NeOAF [18]. These findings support the hypothesis that the systemic inflammatory state can promote and perpetuate AF. However, in our study, slightly higher CRP was observed in patients without NeOAF than in those with NeOAF. It was possibly due to CRP only being measured once at admission, the lack of series follow-up during sepsis, or the development of NeOAF. Therefore, CRP could be influenced by numerous confounding factors during sepsis and we could not demonstrate the positive association between CRP level and NeOAF in our study.

\section{Hypothesis of rhythm control strategy in patients with sepsis and NeOAF}

The development of NeOAF could lead to deteriorated hemodynamic status during sepsis. The occurrence of 
NeOAF makes the disease more complicated and makes treatment during sepsis or septic shock more challenging because of adverse cardiovascular effects including rapid heart rate, irregular rhythm, loss of atrial systole, and neurohormonal activation. In the acute phase, both tachycardia and loss of atrial systole caused by AF could reduce the cardiac output, further destablizing patients with sepsis. Subsequently, the burden of NeOAF in patients with sepsis may result in acute heart failure [35, 36]. Moreover, atrial stasis and sepsis-related coagulopathy could lead to systemic embolization and an increased risk of ischemia stroke [19].

$\mathrm{AF}$ itself has been found to subsequently generate an inflammatory response that further enhances atrial remodeling and perpetuates arrhythmia. Previous studies have proposed that restoration and maintenance of SR in patients with AF could result in gradually decreasing CRP, indicating that AF initiates inflammation [37, 38]. Therefore, inflammation could promote AF and vice versa. To stop the vicious cycle between inflammation and AF, the early management of NeOAF by rhythm control may be beneficial in patients with sepsis.

Our study highlighted that the restoration of SR in patients with sepsis and NeOAF led to more favorable prognosis in comparison with failure to restore SR in these patients. It is evident that restored SR in patients with NeOAF could produce enhanced diastolic filling and a rapid increase in left ventricular systolic performance among patients with NeOAF [39]. Meierhenrich et al. reported that 23 out of 50 surgical patients with septic shock developed NeOAF [18]. They also demonstrated that failure to restore SR was associated with increased ICU mortality (71.4\% versus $21.4 \%$ ). Compared to our study, we conducted a study in a more modest-sized cohort of patients with non-surgical and mixed sepsis and septic shock, and the disease severity of sepsis was relatively lower in our study (SOFA score 7-9 versus 9-12). Consistently, we found that the inhospital mortality rate was significantly higher in the $\mathrm{NeOAF}$ to AF group than in the NeOAF to SR group (61.3\% versus $26.1 \%)$. Restoration of SR appeared to be a favorable prognostic marker of in-hospital mortality in patients with sepsis and $\mathrm{NeOAF}$, and this might have further clinical implications for NeOAF surveillance and treatment in this settings.

\section{Treatment recommendation for NeOAF in patients with sepsis}

So far, recommendations for management of NeOAF in patients with sepsis are dependent on observational studies or expert opinions [40]. In principle, clinicians should manage potentially reversible AF triggers including electrolyte imbalances, rate-affected medication, myocardial injury, and airway obstruction before starting antiarrhythmic treatment.
Pharmacological options between a rate control and a rhythm control strategy remain uncertain in patients with sepsis and NeOAF. The rate control strategy tolerates AF, but improves ventricular filling and avoids a tachycardiainduced cardiomyopathy. It could be achieved by using atrio-ventricular nodal blocking agents, such as betablockers, non-DHP CCBs and digoxin. However, it should be cautiously applied in patients with septic shock because these agents have negative inotropic and vasodilatory effects, probably leading to reduced cardiac output and hypotension. A short-acting beta-blocker with esmolol may be the first consideration in patients with septic shock and NeOAF [41]. In the past, a rhythm control compared with a rate control strategy has shown no survival benefit in non-critically ill patients [42]. Amiodarone can slow nodal conduction and convert AF to sinus rhythm. It has less negative inotropic effects compared with beta-blockers and non-DHP CCB and may be safer in patients with structural heart disease. Nevertheless, current evidence is not strong enough to recommend that amiodarone may improve the prognosis in patients with sepsis and NeOAF [43, 44]. Moreover, as shown in Table 1 , the prevalence of previous treatment with beta-blockers or CCBs was higher in the no NeOAF and NeOAF to SR groups compared to the NEOAF to AF group. However, evidence on NeOAF prophylaxis in patients with sepsis is lacking. Further prospective, randomized studies are necessary to clarify the effects of beta-blockers or CCBs on $\mathrm{NeOAF}$ prophylaxis in patients with sepsis.

Regarding anticoagulant use in patients with sepsis and NeOAF, some studies have demonstrated that $\mathrm{NeOAF}$ is associated with an increased risk of shortterm and long-term ischemic stroke, with threefold higher stroke rates in patients with $\mathrm{NeOAF}$ than in those without NeOAF during sepsis [19, 45]. However, the benefit and risk of anticoagulant use during an acute stage of sepsis remain unclear. Thus, anticoagulants cannot be currently recommended as a cornerstone treatment for sepsis-related $\mathrm{NeOAF}$, and further large prospective studies are warranted.

\section{Study limitations}

The present study had several limitations. First, it was a single-center, retrospective, cohort study that relied on accurate documentation, restricting the external validity of our result. Second, several baseline characteristics of the three groups were not equal. This might lead to bias related to outcomes to a certain extent, despite statistically adjusting for these confounders. Third, we could not determine which factors were the main factors for the restoration of SR in patients with sepsis and NeOAF. This could be attributed to the effect of antiarrhythmic agents, improvement in sepsis-related inflammation, or spontaneous recovery. Fourth, a fixed protocol was not 
used for the management of NeOAF. The pharmacological intervention was retrospectively recorded and based on the clinicians' orders under different clinical conditions. We could not determine which medication led to the therapeutic effect of SR restoration. Fifth, the data on the use of anticoagulation were limited. Sixth, data on the duration of vasopressor use, which may be a confounder in the AF and mortality assessment, were not available in the current study. Seventh, the association between NeOAF to AF or no NeOAF and inhospital mortality was different in the two analysis models. The discrepancy was due to the different definition of outcome in the logistic regression models (event) and Cox proportional hazard models (time to event). Finally, data on long-term follow up in the NeOAF population, such as length of antiarrhythmic therapy, new strokes, and anticoagulant therapy are not obtainable.

\section{Conclusion}

In summary, $\mathrm{NeOAF}$ is prevalent in patients with sepsis and is related to increased in-hospital mortality. We proposed that successful restoration of SR in patients with sepsis and NeOAF may offer a more favorable outcome than in those in whom SR could not be restored. However, a larger, prospective comparative study is needed to elucidate the clinical implications between a rate control and a rhythm control strategy in patients with sepsis and $\mathrm{NeOAF}$.

\section{Key messages}

- New-onset atrial fibrillation (NeOAF) is prevalent and associated with increased mortality in patients with sepsis.

- It remains unclear whether restored sinus rhythm (SR) of NeOAF leads to better outcomes and how to suitably manage in patients with NeOAF.

- NeOAF with failure to restore SR (but not all $\mathrm{NeOAF}$ ) was an independent risk for in-hospital mortality in patients with sepsis.

- Further prospective trials are warranted to elucidate the clinical implications of a rate versus a rhythm control strategy in patients with sepsis and NeOAF.

\section{Additional file}

Additional file 1: Table S1. Hazard ratio of different AF status for in-hospital mortality. Cox proportional hazard model with time-varying exposure was conducted for the analysis. (DOC $32 \mathrm{~kb}$ )

\section{Abbreviations}

AF: atrial fibrillation; APACHE II: Acute Physiology and Chronic Health Evaluation II; CAD: coronary artery disease; CKD: chronic kidney disease; COPD: chronic obstructive pulmonary disease; CPR: C-reactive protein; CVD: cerebrovascular disease; DM: diabetes mellitus; ECG: electrocardiogram;
ICU: intensive care unit; LAD: left atrium diameter; LVEDD: left ventricular end-diastolic dimension; LVEF: left ventricular ejection fraction; LVESD: left ventricular end-systolic dimension; NeOAF: new-onset atrial fibrillation; nonDHP CCB: non-dihydropyridine calcium channel blockers; SOFA: Sequential Organ Failure Assessment; SR: sinus rhythm

\section{Acknowledgements}

None.

\section{Funding}

The study was supported by a grant from the Ministry of National Defense, Republic of China (MAB-105-014).

Availability of data and materials

See Additional file 1.

\section{Authors' contributions}

WSL is the corresponding author and responsible for the concept, revision and approval of this manuscript. JCL and TCL participated in the design of the study and coordination and helped to draft the manuscript. SMC and SPY participated in the sequence alignment and drafted the manuscript. $\mathrm{HBH}$ and CSL performed the statistical analysis and helped to revise the manuscript. WCL, WYL, and WSL were responsible for the data collection and drafted the manuscript. All authors contributed to the data analysis and interpretation. All authors read and approved the final manuscript.

Competing interests

The authors declare that they have no competing interests.

\section{Consent for publication}

All the authors confirmed the manuscript and approved the publication. The corresponding author has completed the "Consent for publication".

\section{Ethical approval and consent to participate}

The institutional review board of Tri-Service General Hospital, National Defense Medical Center approved the study with a protocol number of 2-104-05-003 and informed consent was waived due to the observational design.

\section{Author details}

${ }^{1}$ Division of Cardiology, Department of Internal medicine, Tri-Service General Hospital, National Defense Medical Center, No. 325, Section 2, Cheng-Gong Road, Neihu 114, Taipei, Taiwan. ${ }^{2}$ School of Public Health, National Defense Medical Center, Taipei, Taiwan. ${ }^{3}$ Division of infectious Diseases and Tropical Medicine, Department of Internal medicine, Tri-Service General Hospital, National Defense Medical Center, Taipei, Taiwan.

Received: 4 February 2016 Accepted: 31 October 2016

Published online: 18 November 2016

References

1. Artucio H, Pereira M. Cardiac arrhythmias in critically ill patients: epidemiologic study. Crit Care Med. 1990;18(12):1383-8.

2. Annane D, Sébille V, Duboc D, Le Heuzey JY, Sadoul N, Bouvier E, et al. Incidence and prognosis of sustained arrhythmias in critically ill patients. Am J Respir Crit Care Med. 2008;178(1):20-5.

3. Nisanoglu V, Erdil N, Aldemir M, Ozgur B, Berat Cihan H, Yologlu S, et al. Atrial fibrillation after coronary artery bypass grafting in elderly patients: incidence and risk factor analysis. Thorac Cardiovasc Surg. 2007:55:32-8.

4. Maisel WH, Rawn JD, Stevenson WG. Atrial fibrillation after cardiac surgery. Ann Intern Med. 2001;135:1061-73.

5. Kalavrouziotis D, Buth K, Ali IS. The impact of new-onset atrial fibrillation on in-hospital mortality following cardiac surgery. Chest. 2007;131:833-9.

6. Seguin $P$, Signouret $T$, Laviolle $B$, Branger $B$, Mallédant $Y$. Incidence and risk factors of atrial fibrillation in a surgical intensive care unit. Crit Care Med. 2004:32:722-6.

7. Brathwaite $D$, Weissman $C$. The new onset of atrial arrhythmias following major noncardiothoracic surgery is associated with increased mortality. Chest. 1998;114:462-8.

8. Seguin $\mathrm{P}$, Laviolle B, Maurice A, Leclerca C, Mallédant Y. Atrial fibrillation in trauma patients requiring intensive care. Intensive Care Med. 2006;32(3):398-404. 
9. Hadjizacharia P, O'Keeffe T, Brown CV, Inaba K, Salim A, Chan LS, et al. Incidence, risk factors, and outcomes for atrial arrhythmias in trauma patients. Am Surg. 2011;77(5):634-9.

10. Sander O, Welters ID, Foëx P, Sear JW. Impact of prolonged elevated heart rate on incidence of major cardiac events in critically ill patients with a high risk of cardiac complications. Crit Care Med. 2005;33:81-8.

11. Bone RC, Balk RA, Cerra FB, Dellinger RP, Fein AM, Knaus WA, et al. Definitions for sepsis and organ failure and guidelines for the use of innovative therapies in sepsis. The ACCP/SCCM Consensus Conference Committee. American College of Chest Physicians/Society of Critical Care Medicine. Chest. 1992;101(6):1644-55.

12. Dellinger RP, Levy MM, Rhodes A, Annane D, Gerlach H, Opal SM, et al. Surviving Sepsis Campaign: international guidelines for management of severe sepsis and septic shock: 2012. Crit Care Med. 2013;41(2):580-637.

13. Singer M, Deutschman CS, Seymour CW, Shankar-Hari M, Annane D, Bauer $M$, et al. The Third International Consensus Definitions for Sepsis and Septic Shock (Sepsis-3). JAMA. 2016;315(8):801-10.

14. Arora S, Lang I, Nayyar V, Stachowski E, Ross DL. Atrial fibrillation in a tertiary care multidisciplinary intensive care unit-incidence and risk factors. Anaesth Intensive Care. 2007;35:707-13.

15. Goodman S, Shirov T, Weissman C. Supraventricular arrhythmias in intensive care unit patients: short and long-term consequences. Anesth Analg. 2007;104:880-6.

16. Christian SA, Schorr C, Ferchau L, Jarbrink ME, Parrillo JE, Gerber DR. Clinical characteristics and outcomes of septic patients with new-onset atrial fibrillation. J Crit Care. 2008:23:532-6.

17. Salman S, Bajwa A, Gajic O, Afessa B. Paroxysmal atrial fibrillation in critically ill patients with sepsis. J Intensive Care Med. 2008;23(3):178-83.

18. Meierhenrich R, Steinhilber E, Eggermann C, Weiss M, Voglic S, Bögelein D, et al. Incidence and prognostic impact of new-onset atrial fibrillation in patients with septic shock: a prospective observational study. Crit Care. 2010;14:R108.

19. Walkey AJ, Wiener RS, Ghobrial JM, Curtis LH, Benjamin EJ. Incident stroke and mortality associated with new-onset atrial fibrillation in patients hospitalized with severe sepsis. JAMA. 2011;306(20):2248-54.

20. Walkey AJ, Greiner MA, Heckbert SR, Jensen PN, Piccini JP, Sinner MF, et al, Atrial fibrillation among Medicare beneficiaries hospitalized with sepsis: incidence and risk factors. Am Heart J. 2013;165(6):949-55.

21. Chen AY, Sokol SS, Kress JP, Lat I. New-onset atrial fibrillation is an independent predictor of mortality in medical intensive care unit patients. Ann Pharmacother. 2015:49(5):523-7.

22. Kuipers S, Klein Klouwenberg PM, Cremer OL. Incidence, risk factors and outcomes of new-onset atrial fibrillation in patients with sepsis: a systematic review. Crit Care. 2014;18(6):688.

23. Gandhi S, Litt D, Narula N. New-onset atrial fibrillation in sepsis is associated with increased morbidity and mortality. Neth Heart J. 2015;23(2):82-8.

24. Shaver CM, Chen W, Janz DR, May AK, Darbar D, Bernard GR, et al. Atrial fibrillation is an independent predictor of mortality in critically ill patients. Crit Care Med. 2015:43(10):2104-11.

25. Knaus WA, Draper EA, Wagner DP, Zimmerman JE. APACHE II: a severity of disease classification system. Crit Care Med. 1985;13(10):818-29.

26. Vincent $J$, de Mendonça A, Cantraine F, Moreno R, Takala J, Suter PM, et al. Use of the SOFA score to assess the incidence of organ dysfunction/failure in intensive care units: results of a multicenter, prospective study. Working group on "sepsis-related problems" of the European Society of Intensive Care Medicine. Crit Care Med. 1998;26:1793-800.

27. Szklo M, Nieto FJ. Epidemiology: Beyond the basics. 2nd ed. Sudbury: Jones and Bartlett Publishers; 2007.

28. Issac TT, Dokainish H, Lakkis NM. Role of inflammation in initiation and perpetuation of atrial fibrillation: a systematic review of the published data. J Am Coll Cardiol. 2007:50(21):2021-8.

29. Hu YF, Chen YJ, Lin YJ, Chen SA. Inflammation and the pathogenesis of atrial fibrillation. Nat Rev Cardiol. 2015;12:230-43.

30. Brown AO, Millett ER, Quint JK, Orihuela CJ. Cardiotoxicity during invasive pneumococcal disease. Am J Respir Crit Care Med. 2015;191:739-45.

31. Brown SM, Tate Q, Jones JP, Knox DB, Kuttler KG, Lanspa M, et al. Initial fractal exponent of heart rate variability is associated with success of early resuscitation in patients with severe sepsis or septic shock: a prospective cohort study. J Crit Care. 2013;28:959-63.

32. Rudiger A, Singer M. Mechanisms of sepsis-induced cardiac dysfunction. Crit Care Med. 2007;35:1599-608.
33. De Backer D, Biston P, Devriendt J, Madl C, Chochrad D, Aldecoa C, et al. Comparison of dopamine and norepinephrine in the treatment of shock. $\mathrm{N}$ Engl J Med. 2010;362:779-89.

34. Aviles RJ, Martin DO, Apperson-Hansen C, Houghtaling PL, Rautaharju P, Kronmal RA, et al. Inflammation as a risk factor for atrial fibrillation. Circulation. 2003;108(24):3006-10

35. Walkey AJ, Ambrus D, Benjamin EJ. The role of arrhythmias in defining cardiac dysfunction during sepsis. Am J Respir Crit Care Med. 2013;188(6):751.

36. Wang TJ, Larson MG, Levy D, Vasan RS, Leip EP, Wolf PA, et al. Temporal relations of atrial fibrillation and congestive heart failure and their joint influence on mortality: the Framingham Heart Study. Circulation. 2003; 107(23):2920-5.

37. Marcus GM, Smith LM, Glidden DV, Wilson E, McCabe JM, Whiteman D, et al. Markers of inflammation before and after curative ablation of atrial flutter. Heart Rhythm. 2008:5(2):215-21.

38. Kallergis EM, Manios EG, Kanoupakis EM, Mavrakis HE, Kolyvaki SG, Lyrarakis $\mathrm{GM}$, et al. The role of the postcardioversion time course of hs-CRP levels in clarifying the relationship between inflammation and persistence of atrial fibrillation. Heart. 2008;94(2):200-4.

39. Viswanathan K, Daniak SM, Salomone K, Kiely T, Patel U, Converso K, et al. Effect of cardioversion of atrial fibrillation on improvement in left ventricular performance. Am J Cardiol. 2001:88(4):439-41.

40. Walkey AJ, Hogarth DK, Lip GY. Optimizing atrial fibrillation management: from ICU and beyond. Chest. 2015;148(4):859-64.

41. Morelli A, Ertmer C, Westphal M, Rehberg S, Kampmeier T, Ligges S, et al. Effect of heart rate control with esmolol on hemodynamic and clinical outcomes in patients with septic shock: a randomized clinical trial. JAMA. 2013;310(16):1683-91.

42. Wyse DG, Waldo AL, DiMarco JP, Domanski MJ, Rosenberg Y, Schron EB, et al. A comparison of rate control and rhythm control in patients with atrial fibrillation. N Engl J Med. 2002;347(23):1825-33.

43. Delle Karth G, Geppert A, Neunteufl T, Priglinger U, Haumer M, Gschwandtner $M$, et al. Amiodarone versus diltiazem for rate control in critically ill patients with atrial tachyarrhythmias. Crit Care Med. 2001;29(6):1149-53.

44. Hou ZY, Chang MS, Chen CY, Tu MS, Lin SL, Chiang HT, et al. Acute treatment of recent-onset atrial fibrillation and flutter with a tailored dosing regimen of intravenous amiodarone. A randomized, digoxin-controlled study. Eur Heart J. 1995;16(4):521-8.

45. Walkey AJ, Hammill BG, Curtis LH, Benjamin EJ. Long-term outcomes following development of new-onset atrial fibrillation during sepsis. Chest. 2014;146(5):1187-95.

\section{Submit your next manuscript to BioMed Central and we will help you at every step:}

- We accept pre-submission inquiries

- Our selector tool helps you to find the most relevant journal

- We provide round the clock customer support

- Convenient online submission

- Thorough peer review

- Inclusion in PubMed and all major indexing services

- Maximum visibility for your research

Submit your manuscript at www.biomedcentral.com/submit 